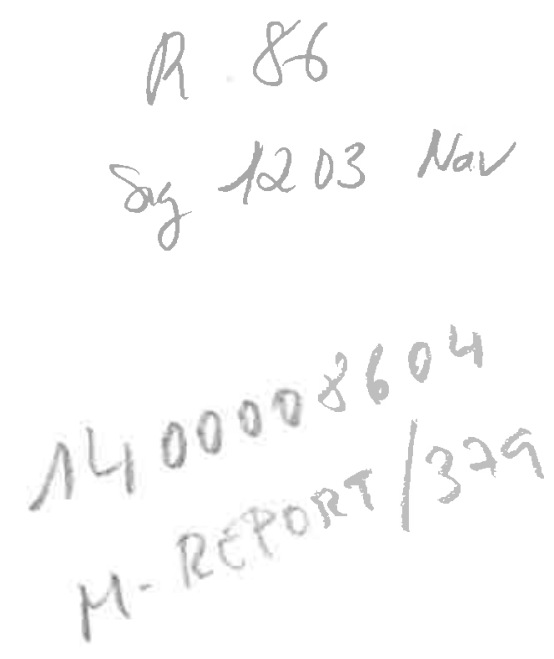

SYSTOLIC IMPLEMENTATION FOR

DECONVOLUTION ITERATIVE

ALGORITM

J.J. NAVARRO GUERRERO

V. CASARES GINER

RR85/19 


\section{RESUMEN.}

Para resolver algunos algoritmos de Procesado Digital de Señal donde se requiere gran velocidad de cálculo son necesarias arquitecturas de propósito especial con procesado paralelo. Los procesadores systólicos son buenos candidatos para implementar estas aplicaciones. En este trabajo se presenta una implementación systólica de un algoritmo iterativo de deconvolución. Un array bidimensional implementa una iteración del algoritmo. Este módulo básičo puede concatenarse repetidamente de forma que permite su uso en aplicaciones en tiempo real.

\section{ABSTRACT.}

Systolic architectures implement regular algorithms in hardware, in, order to obtain high computational throughput. In this paper we provide a modular architecture for a deconvolution iterative algorithm. The basic module is a systolic array which implement one iteration of the algorithm recently proposed in 1 . The algorithm is a generalization of the method to invert non singular polynomial transfer function, previously published in 2 . The basic systolic module can be repeatedly concatenated in such a way that can be used in real time applications. 


\title{
SYSTOLIC IMPLEMENTATION FOR DECONVOLUTION ITERATIVE ALGORITHM
}

\author{
Juan José Navarro-Guerrero * \\ Vicente Casares-Giner ** \\ * Facultad de Informática (U.P.C.) \\ * E.T.S.I. Telecomunicación (U.P.C.)
}

c/Jordi Girona Salgado $\mathrm{s} / \mathrm{n} .08034$ Barcelona, (Spain)

\section{ABSTRACT}

Systolic architectures 1mplement regular algorithms in hardware, in order to obtais high computational throughput. In this paper we provide a modular architectu re for a deconvolution iterative algorithm. The basic module is a systolic array which implement one iteration of the algorithm recently proposed in $|1|$. The algorithm is a generalization of the method to invert non singular polynomial transfer function, previously publisined in | 2 ! . The basic sys tolic module can be repeatedly concatenated in such a way that can be used in zeal time applications.

\section{INTRODUCTION}

Special purpose system with parallel processing can be used for some Digital Signal Processing when high-speed processing is required. Systoiic Array processors allow to obtain high computational througinput for reasonable I/O requirements when it is possible to perform repeated calculations on each data iten for one of more large input data streans. The simplicity and the regularicy of Processing Elements (PES), and thetr interconexions, as soon as the locality for communications, make this architectures very suitables for very LeIg Scale Integrations (VLSI) $|7|,|3|,|8|$.

Recursive anc local data-depencient algorithms are good candidates to be imple ment with systolic arahiteceures. This is the case of a deconvolution algorithm we present here, for which, we provice a systoilc realization. Is the next lines we ir troduce very briefly, the convolution and decosvolution problems.

There are some applications where the recorded information has been previous ly cegraded. One of the most common model used, consist of noise added to the signal

This work was supported in part by CRICr? under grant no 2906-83. which has been distorted by an invariant linear system, that is

$y=h * x+n$

In (1), $x$ denotes the useful information, I the random noise, $b$ the deterministic im pulse response of the linear system and $y$ the avallable information (* is the convolution operatar). The deconvolution problem consist of recovering the information $x$, for a given degrading system $h$, a given random process $n$ and a recorded informstion $y$.

This paper has two main parts. In the first one we present an irerative algo rithm dealing with the inversion of (1) in a digital fashion. In addition, we assume that the transfer function of $h$ is defined by a finite impulse response (FIR), free of zeros on the unit cifcumference -4 . Also, in (1) we have considered $n=0$. Under such assumptions, for instance, we can always factorize the transfer function in a product of two factors of finite order, ha ving their zeros inside and outside of ?. This allow us to implement the inversion as a cascade of two systems of minimum and maximum phases 19 . However, if we are interested in a real time processing this me thod is not longer valid. The algorithm we present here, overcomes this dexiciency.

The second part of the paper, deais with systolic architectires fur the algorithm. In the literature, some systoile 1m plementations has been proposed in the deconvolution area 5 !. FOr instance, G.I. $I \dot{i}$ and $B . W$. Wat $6 \mid$ provide a realizatior for the case that ali zeros of the trans. fer function of h lie insiae $T$. This and other cases, as we will see, can be obtained as particularizations of our systolic architecture.

\section{THE GENERAI ALGORITEM}

Let $\mathrm{E}_{M}\left(\mathrm{z}^{-1}\right)$ be the transfer function of the degrading system, which consist of a real polynomial in $z^{-i}$ of degree $M$, and it is free of zeros on $i$. Let $R(0 \leqslant R \leqslant M)$ be the number of zoots outsioe - . It is weil 
known that, in general, the ldeal inverse is stabie but not causal. So, if we want to implement the irverse of $\left(h^{-1}\right)$ in a real time environment, we need to approximate the ideal impulse response by a causal one. For that purpose, in $|1|$ it was presented a decomposition of $\mathrm{B}_{\mathrm{M}}\left(z^{-1}\right)$ in or der to carfy out the refered real time inversion. Here, and for the sake of comprehensibility, we will repeat the basic argu ments of $|1|$. nomial:

Given $\mathrm{E}_{\mathrm{M}}\left(\mathrm{z}^{-1}\right)$ we look for a $2 \mathrm{D}$ poly-

$\operatorname{HP}\left(z^{-1}, W^{-1}\right)=D_{M}\left(z^{-1}\right)+\sum_{i=1}^{T} N_{M 1}\left(z^{-1}\right) w^{-1}$

where $D_{Y}\left(z^{-1}\right)$ and $M_{Y i}\left(z^{-1}\right)(1,2, \ldots, T)$ are polynomials of degrể $M$. Besides, the conditions we want to be satisfied are:

(2). $-\mathrm{a}: \operatorname{EP}\left(z^{-1}, 1\right)=E_{M}\left(z^{-1}\right)=m_{0} h_{m} z^{-m}$

(2) $-b: F\left(z^{-1}\right)=z^{P} D_{M}\left(z^{-1}\right)$ be a minimum phase factor, i.e., Mall zeros and poles insi de $T$. P is a positive constant to be de termined.

(2) $-c: \operatorname{EP}\left(\exp (j \omega), \mathrm{W}^{-1}\right)$ be a minimum phase. factor with the variable $W$ for any is $g$ i ven.

From condition (2)-a, the sum of coer ficlents $\left\{d_{m}\right\}$ and $\left\{n_{i m}\right\} \quad(i=1,2, \ldots, T)$ with the same power of $z^{-1}$, must be equal to the associated coefficient of $\mathrm{H}_{M}\left(z^{-1}\right)$, that is,

$\mathrm{n}_{\mathrm{m}}=\mathrm{d}_{\mathrm{m}}+\underset{1=1}{\frac{T}{2} n_{1 m} ; \quad 0 \leq m \leq M}$

From the condition $(2)^{-b}$ is not difficult to see that

$d_{m}=0, d_{\mathrm{p}} \neq 0 ; 0 \leq m<p$

About the positive integer number $D$, we can very easy show from condition (2)-c that it must be equal to the number of roots of $\mathrm{B}_{M}\left(z^{-1}\right)$ outside $\Gamma ; 1 . e ., P=R$. In or der to proof these, we write (2) as:

$\operatorname{EP}\left(z^{-1}, W^{-1}\right)=$

$=D_{M}\left(z^{-1}\right)\left(i-W_{1}\left(z^{-1}\right) / W\right) \ldots\left(1-W_{T}\left(z^{-1}\right) / W\right)$

,i.e., a Eactorial decomposition of

$\operatorname{HP}\left(z^{-1}, w^{-1}\right)$ in the variable $W^{-1}$, where the roots $w_{i}\left(z^{-1}\right)$ are functions of $z$. Particulary, when $z=\exp (j w)$, and according to

$(2)-c, \mid W_{i}(\exp (-j w))^{\prime}<1$ for $i=1,2, \ldots, T$.

Then, when varies from 0 to $2 \pi$ the argument of $\operatorname{HP}(\exp (j \omega), 1), i . e .$, of

$H_{M}(\exp (-j \omega))$, varies from 0 to $-2 R \pi$ which forces

$D_{M}\left(z^{-1}\right)=\sum_{m=R}^{M} d_{m} z^{-m}$

that is, $\mathrm{P}=\mathrm{R}$. Figure 1-a gives the arzay support of the proposed decomposition.

If the decomposition (2) exist, the solution to the convolution equation

$y_{m}=\sum_{i=0^{n}}^{M} x_{m-i}$ (or $\left.Y\left(z^{-1}\right)=E_{M}\left(z^{-1}\right) X\left(z^{-1}\right)\right)$

can be carried out by the following iterative algorithm in the frequency domain:

$x^{p}\left(z^{-1}\right)=$

$=\left[Y\left(z^{-1}\right)-\sum_{i=1}^{N_{M i}}\left(z^{-1}\right) \cdot x^{p-1}\left(z^{-1}\right)\right] / D_{M}\left(z^{-1}\right)$

, where $x^{p}\left(z^{-1}\right)$ denotes the p-th estimate of $x\left(z^{-1}\right)$ obtained in the p-th iteration. The formulation of (8) in the time domain is given by:

$x_{m-R}^{P}=\frac{1}{a_{R}}\left[y_{m}-\sum_{j=R+1}^{M} a_{j} x_{m-j}^{P} \sum_{i=1}^{T} \sum_{j=0}^{M} n_{i j} x_{m-j}^{P-i}\right]$ , where $x^{P}$ denotes the p-th estimate of the sample $x_{m}^{m}$ obtained in the p-th iteration.

A simple and general decomposition

Here, and in order to provide a systolic architecture, we show again a trivial decomposition according to (2), which was already presented in $|1|$. The mentioned decomposition can be obtained from the Algebra Fundamental Theorem. Let $\mathrm{E}_{+}\left(z^{-1}\right)$ and $\mathrm{H}_{\text {_ }}\left(z^{-1}\right)$ be the two factor of $\mathrm{E}_{\mathrm{M}}\left(z^{-i}\right)$ where the first one contain the $M-B$ roots inside $r$ and the second one the $R$ roots outside $\Gamma:$

$$
\begin{aligned}
H_{M}\left(z^{-1}\right) & =H_{+}\left(z^{-1}\right) E_{-}\left(z^{-1}\right)= \\
& =\sum_{i=0}^{M-R} h_{+i} z^{-1} \sum_{i=0}^{R} h-1 z^{-i}
\end{aligned}
$$

If we identify

$$
\begin{aligned}
D_{M}\left(z^{-1}\right) & =h_{-} z^{-R} H_{+}\left(z^{-1}\right) \\
N_{M 1}\left(z^{-1}\right) & =h_{-i} z^{i-R} H_{+}\left(z^{-1}\right) \\
i & =1,2, \ldots, T=R
\end{aligned}
$$

it is clear that ( $($ ) is a valid solution sa tisfying (2). In figure 1 bo we represent the array support for this simple and exact

\begin{tabular}{|l|l|l|l|l|}
\hline$n_{3,0}$ & $n_{3,1}$ & $n_{3,2}$ & $n_{3,3}$ & $n_{3,4}$ \\
\hline$n_{2,0}$ & $n_{2,1}$ & $n_{2,2}$ & $n_{2,3}$ & $n_{2,4}$ \\
\hline$n_{1,0}$ & $n_{1,1}$ & $n_{1,2}$ & $n_{1,3}$ & $n_{1,4}$ \\
\hline \multicolumn{6}{|c|}{} & $d_{2}$ & $d_{3}$ & $d_{4}$ \\
\hline
\end{tabular}

Fig. 1.a: General decompusition. $M=4, R=2, T=3$.

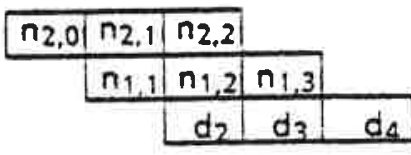

\begin{tabular}{|l|l|l|l|l|}
\hline$h_{0}$ & $h_{1}$ & $h_{2}$ & $h_{3}$ & $h_{4}$ \\
\hline
\end{tabular}

Fig. 1.b: Simple decomposition $M=4, R=T=2$. 
decomposition. In this particular decomposition the time aomain formulation of (9) becomes as:

$x_{m-R}^{P}-\frac{1}{d_{R}}\left[y_{m}-\sum_{j=\frac{R}{L}+1}^{M} d_{j} x_{m-j}^{P}-\sum_{i=1}^{R} \sum_{j=R-i}^{M-i} n_{1 j} x_{m-j}^{p-i}\right]$

\section{SYSTOLIC ARCEITECTURES}

In this section we propose a systo11c architecture for (12). Figure 2-c shows the $\hat{i}$-Dimensional systolic array which compute one iteration $\left\{x^{9}\right\}$ we will name this systolic array, as a basic module; and its PEs are labeled as $P E(i, j)$, $i=0,1, \ldots, R_{j} j=0,1, \ldots . M-R$. Three different kinds oI PES can be differentiated in the array; type A, B and $C$, (Eigure 2-b). Sub index sets $(1=0,1, \ldots, R-1 ; j=0,1, \ldots, M-R)$ $(i=R, j=1,2, \ldots, M-R)$ and $(i=R, j=0)$ define the PES of type A, B ard C respectively.

In order to explain the computing spatial distribution in the array, we will use the expresion (12) transformated after some manipulations in:

$x_{m}^{P}=\frac{1}{d_{R}}\left\{y_{m+R}-\sum_{j=1}^{M-R}\left[a_{R+j} x_{m-j}^{P}-\right.\right.$

$\left.\left.-\sum_{i=0}^{R-1} n_{R-i, 1+j} x_{m+R-i-j}^{P-R+i}\right]-\sum_{i=0}^{R-1} n_{R-1,1} x_{m+R-i}^{P-R+1}\right\}$

Each PE $(i, j)$ of type A compute the expression

$\alpha_{i j}=\alpha_{i-1, j} n_{R-i, i+j} x_{m+R-i-j}^{p-R+i}$

$\{0\}\{0\}\{0\}\{0\}\{0\}\{0\}$

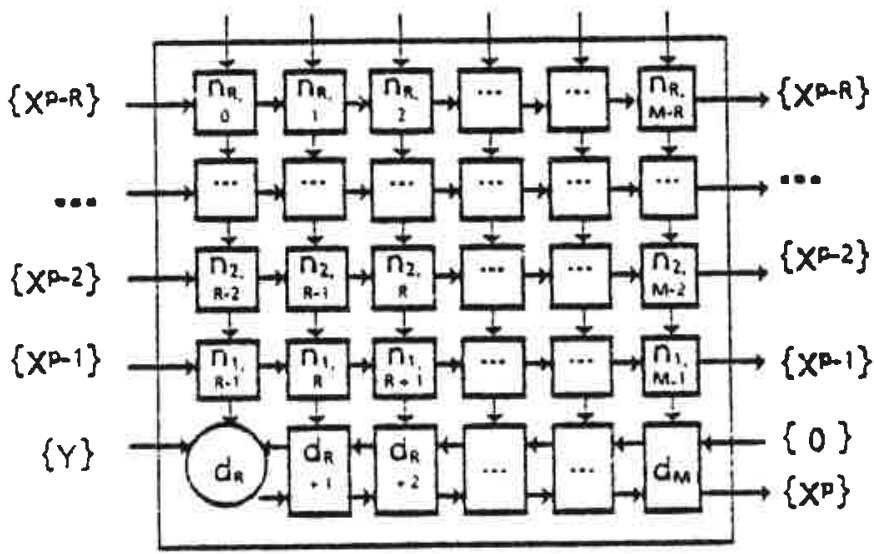

in such a way that the partial result ob tained for each PEs column of type $A$ is $a_{j}=\sum_{i=0}^{R=1} n_{R-i, j+j} x_{m+R-i-j}^{p-R+i}$

Each PE $(1, j)$ of type B compute the expression

$3_{j}=B_{j+i}+a_{R+j} x_{m-j}^{D}+\alpha j$

in such a way that the DEs zow of type $B$ compute the expression:

$s=\sum_{j=1}^{M-R}\left[d_{R+j} x_{m-j}^{P}+\sum_{i=0}^{R-1} \Omega_{R-i, i+j} x_{m+R-i-j}^{P-R+i}\right]$

The PE $(R, 0)$ of type $C$ compute the desired result

$x_{\text {II }}^{p}=\frac{1}{d_{R}}\left[y_{m+R}-\hat{s-\alpha} 0\right]$

In order to compute $(14)-(18)$ cor rectly, the input data streams must be in jected into the basic module with an empty clock cicle between consecutive data samples. Elgure 3 shows the I/O data systems for $M=7$ and $R=3$.

If we want to obtain several seouen ces $\{x p(p=1,2, \ldots$.$) , we concatenate as$ many basic modules as number of 1terations are desired. Figure 4 give us the interconection topology for 5 basic modules. Notice that this configuration performs a real time deconvolution: where the initial conditions are $\left\{x^{\circ}\right\}=\left\{x^{-1}\right\}=$ $=\left\{x^{-2}\right\}=\{y\}$.

When $R=0$ the deconvolution algorithm is only recursive, not iterative, since, and according to the general decom position $(2), a_{m}=h_{m} m=0,1, \ldots, M$. Consequently our algorithm becames the known method of successive substitutions. The fesulting basic module has only PEs of ty pe $B$ and $C$, which is similar to the arch $i$ tecture proposed in $|6|$.

\section{CONCIUSIONS}

This paper concerrs witi a systolic architecture sor a deconvolution iterative

Fig. 2.a: Basic Module.

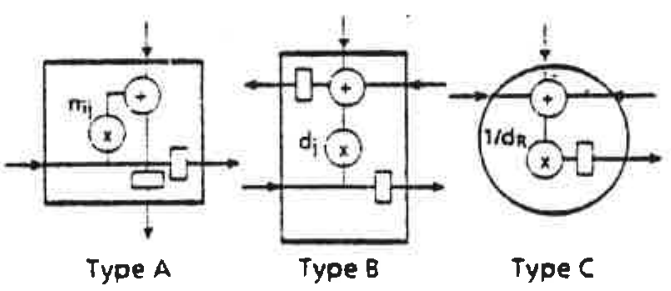

Fig. 2.b: Processing Elements.

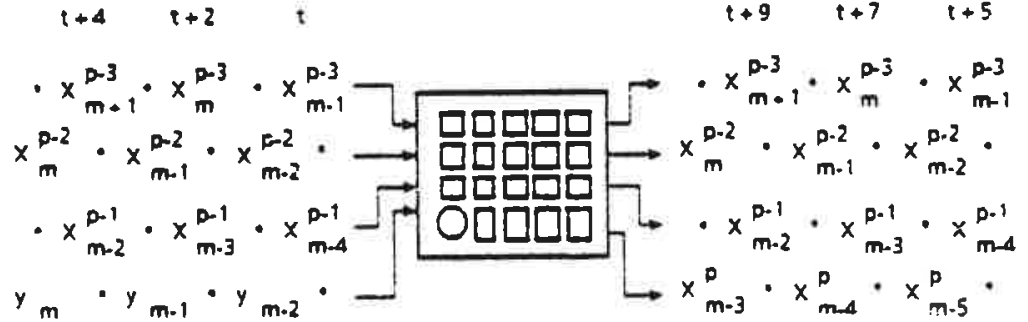

Fig. 3: Input/Output data flow. 
[y]

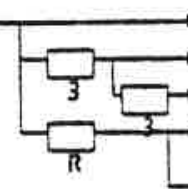

Delay etements

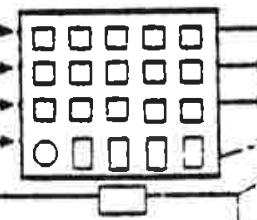

$M \cdot R+4$

Fig. 4: Concatenation of Basic Modules.

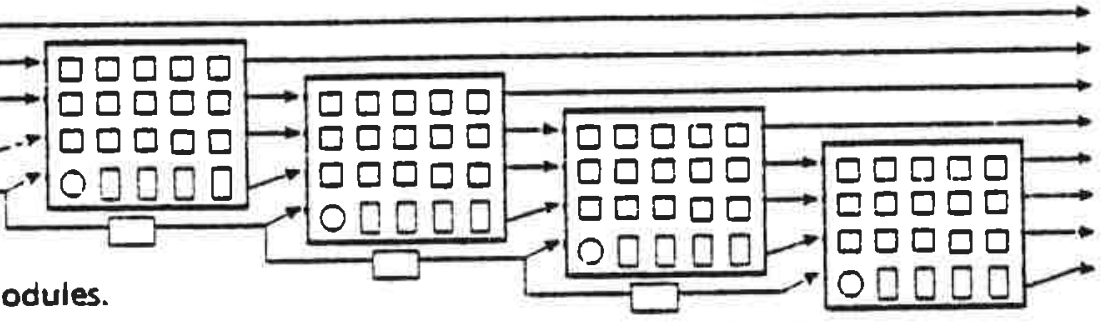

$\{x-2\}$

$[x-1\}$

algorithm. He have presented a modular configuration such that each basic module performs one iteration of the algorithm. W1th repeated concatenation of that basic moduies we can obtain a decon volution system for real time applications.

Further studies are needed in order to improve the efficiency of this and others systolic architectures. In that sense, the algorithms expresed by (12) and is $|2|$ has been stucted in $|10|$.

\section{REFERENCES}

1) V. Casares-G1ner, "Implementation of $i-D$ and $2-D$ Digital Filters by Iterative and Recursive Methods". Proceedings of the International Conference on Digital Signal Processing, pp. 18-21. Florence. September 1984.

| 2! V. Casares-Giner et al., "Asymptotical inversion of non-singular digital symmetrical polynomial transfer functions". Signal Processing, vol. 4, pp. 5-16, January 1982.

| 3 H.I. Rung, "Why Systolic architectures?". I.E.E.E. Trans. on Computers. Vol. C-15, po. 37-46, January 1982.

4 A.V. Oppenhein \& R.W. Schafier, "Digital Signal Processing". Prentice Hall 1978 .

5 : R. Yoho \& D. Preis, "Systolic Architectures for Deconvolution Algorithms". Proceedings of the ICASSF1984 , paper 25A.3.1-25A.3.4.

16 G.J. Ii and B.W. Wah, "The Design of Optimal Systolic Arrays". I.E.E.S. rrans. on Computers, vol. C-34, op. 56-67, January 1985 .

7 घ.T. Kung \& C.E. Leiserson, "Systolic Arrays (for VLSI)". Sparse Matrix Proceedings 1978 , Duff, I.S. and Stewart, G.W., eds. Society for Incustrial and Applied Mathematics, pp. 256-282, $1979^{\circ}$.

| $8 \mid$ C. Mead \& L. Conway, "Introducsion to VLSI Systems". Addison-Wesley 1980 .

9 T.B. Euang, "Stabllity of two-dimensional recursive Eilters". I.E.E.E. Trans. Audio Electzoacoust. Vol. 20, po. 158-163. June 1972 .
! 10 ! J.J. Navarro-Guerrero \& V. CasaresGiner, "Improvement of the eficlency in deconvolution Systolic Architectu res". Submited to tie 20 simposio de Electronica das Telecomunicaçoes. Fo zum Piscoas, Lisboa, 21,22 e, $23 \mathrm{de}^{-}$ Maio de 1986. 